\title{
Capturing and Sharing Human Digital Memories with the Aid of Ubiquitous Peer- to-Peer Mobile Services
}

\author{
Chelsea Dobbins, Madjid Merabti, Paul Fergus, David Llewellyn-Jones \\ School of Computing and Mathematical Sciences, Liverpool John Moores University \\ Liverpool, UK \\ C.M.Dobbins@2006.ljmu.ac.uk, \{M.Merabti, P.Fergus, D.Llewellyn-Jones\} @1jmu.ac.uk
}

\begin{abstract}
The explosion of mobile computing and the sharing of content ubiquitously has enabled users to create and share memories instantly. Access to different data sources, such as location, movement, and physiology, has helped to create a data rich society where new and enhanced memories will form part of everyday life. Peer-to-Peer (P2P) systems have also increased in popularity over the years, due to their ad hoc and decentralized nature. Mobile devices are "smarter" and are increasingly becoming part of P2P systems; opening up a whole new dimension for capturing, sharing and interacting with enhanced human digital memories. This will require original and novel platforms that automatically compose data sources from ubiquitous ad-hoc services that are prevalent within the environments we occupy. This is important for a number of reasons. Firstly, it will allow digital memories to be created that include richer information, such as how you felt when the memory was created and how you made others feel. Secondly, it provides a set of core services that can more easily manage and incorporate new sources as and when you are available. In this way memories created in the same location, and time are not necessarily similar - it depends on the data sources that are accessible. This paper presents DigMem, the initial prototype that is being developed to utilize distributed mobile services. DigMem captures and shares human digital memories, in a ubiquitous P2P environment. We present a case study to validate the implementation and evaluate the applicability of the approach.
\end{abstract}

Index Terms - Ubiquitous Computing; Ad-Hoc Networking; Mobile Services; Lifelogging; Human Digital Memory; Peerto-Peer Network

\section{INTRODUCTION}

Memories are those precious moments that stay with you for a lifetime and are composed of not only the people you interact with and the places you go, but also the feeling that encapsulates those events. They are a significant part of our existence. Reliving those past experiences, through reminiscing, is a practice that is a substantial part of our lives, and one that has been performed over thousands of years. As technology advances, preserving and sharing our entire lives in a digital format is slowly becoming feasible. The explosion of mobile computing and ubiquitous content sharing has enabled users to create and share memories instantly. The utilization of social-networking technologies has created a society where sharing is part of everyday life. However, the weakness of sharing content in a client-server environment, like the web, is that the core functionalities of storing and distributing data to clients depends solely on a single entity, the server. Therefore, if the server malfunctions then the system is essentially rendered useless. An alternative approach worth considering is peer-to-peer (P2P). Parameswaran et al. [1] describe this approach as all nodes being equal in their capacity for sharing information with other network members.

In terms of capturing life memories, combining mobile computing and peer-to-peer networking technologies is the quickest, easiest and most popular way to share memories. With $91 \%$ of adults, in the UK, owning a mobile phone [2], as well as 365.4 million phones being shipping in the second quarter of 2011 [3], they have become a constant fixture in our lives. As their popularity increases, clearly these intelligent devices will be the primary tool used for capturing memories. In terms of the platform to develop this interactive process Android is a popular choice because it is open source. It is compatible with most devices and provides developers with full access to the same framework APIs used by the core applications. Developers, furthermore, have the ability to take advantage of the device's hardware [4]. Another advantage of using Android is that it is compatible with Java and is also capable of utilizing P2P network technologies.

Initial work focused on the idea of creating rich interactive digital memories. The memories contain vivid structures and varied information sources that emerge through the semantic clustering of content and other memories. This technique is particularly useful for developing compositions between other memories about ourselves, our friends and our family [5]. In order for this idea to be realised all of this data needs to be grouped together to form a "complete memory". This has led to the development of creating memory "boxes". These "boxes" will be composed of a variety of data, from ubiquitous sources, and are then catalogued and organised so that similar memories are clustered together. Memories are linked between streams that in turn are linked to our associate's memories. This has the benefit of creating endless streams or clusters of memories that can be reasoned over and reflected upon. As our human memories develop and grow so will their digital counterpart. In developing this idea P2P technologies [6], ported with the Android platform [7] have been utilized. Thus creating a virtual network where peers can construct their memories, through accessing 
C. Dobbins, M. Merabti, P. Fergus, and D. Llewellyn-Jones, "Capturing and Sharing Human Digital Memories with the Aid of Ubiquitous Peer-to-Peer Mobile Services," in The 10th IEEE International Conference on Pervasive Computing and Communications (PerCom'12), 2012, pp. 64-69.

distributed services, as we will see in the remainder of this paper.

\section{RELATED WORK}

The mobile era is upon us and with these devices increasing in sophistication and decreasing in size, they are the ideal candidate to capture and share life memories. Memories are often impulsive events and are better suited to being captured and shared on a portable device. These devices are adequately compact to be carried around and are sophisticated enough for sharing content amongst users. Olsson et al. [8] reiterates this point by stating that "Mobile phones offer natural opportunities for collecting instant digital pictures and videos because of their immediate availability to users". Mobile computing, along with peerto-peer networking and the notion of collecting digital life memories, has begun to generate a great deal of interest. Furthermore, an extensive amount can be learnt from existing work in this area.

One such approach is JMobiPeer, a P2P middleware for mobile ad hoc networks (MANETs). It has been designed to work on J2ME enabled portable devices and is compatible with the JXTA peer-to-peer protocols [9]. The JMobiPeer system has obtained good results, in relation to the discovery time and bytes exchanged. However, as Wang and Motzfeldt [10] observe, it "has only been tested on emulators on standard PCs. This is likely due to high requirements on CPU and memory from running the framework." Therefore, real-world testing on actual mobile devices would be required to determine whether the application could be executed on devices that are far less capable.

Taking an opposing view, Tsai et al. [11] created a Mobile Social Software (MoSoSo) application that runs on mobile devices. The application "allows users to discover, communicate and share resources with each other". It is a P2P social networking application in which users can view friends, share files, message each other, add new friends and edit their own profiles. The MoSoSo application has some interesting features that could be exploited within the system posited in this paper. In particular, the ability to transfer picture files over its $\mathrm{P} 2 \mathrm{P}$ platform is quite fascinating and one that is being explored.

Meanwhile, Palazzi et al. [12] created the P2PBluetooth platform, a "proof-of-concept file sharing application for mobile phones that works through Bluetooth connectivity" [12]. In particular, their work focuses on creating a P2P network using J2ME. An interesting aspect to their work is file sharing and the idea of proximity. Proximity is one of the features discussed in previous work [5] and is an idea that will be expanded upon. In relation to sharing memories, particularly at events, proximity is important when sharing with people in the direct vicinity. However, this work is limited to very short distances, since Bluetooth has a limited range. Our work aims to be less restrictive, by creating connections using JXTA to support different proximity requirements.

In other works, Ismail et al. [13] have designed a framework to identify personal memories, through photo image analysis and a reporting system. Their system uses the JXTA P2P networking architecture to create a virtual network of peers who share their serendipitous moments among themselves [14]. This work is particularly interesting as JXTA is a platform that is currently being explored for the system.

Clearly mobile computing and peer-to-peer networking technologies are becoming increasingly more popular and important. These tools and standards provide mechanisms for interconnecting device functionality as independent network discoverable services. However, this alone does not support the memory structures required. There is a need to build additional middleware services to achieve this, and this will be the focus of future work. Nonetheless, this paper describes the foundation services required to deploy and access any number of data sources that would be useful in creating rich digital life memories. In this way, the services platform provides a plug and play system for memory data sources that can be exploited by any digital life memory middleware services, as we will see in the remainder of this paper.

\section{P2P MOBILE SERVICES PlATFORM}

In order to explore the idea of creating memory boxes the system being considered obtains data from a variety of pervasive devices, within the P2P network. Initial design focuses on the user wanting to create a memory on a desktop PC; however, their device does not have the necessary hardware to facilitate this request. For example, the user wants to capture photos and the PC doesn't have a portable camera to do this. In this instance, a request is made to all peers within the network, in order to obtain the use of their camera. The mobile client responds to this request and connects to the PC. The connected device then captures the desired data and sends it back to the PC client. In order to connect the peers together a P2P network would need to be implemented.

For initial purposes, the scenario will be composed of three peers (PC and mobile peers), and a rendezvous peer. The rendezvous peer will act as an assembly point where the other peers will meet. Once this has been completed the lines of communication between the peers has been opened. Communication occurs via bidirectional pipes. The mobile peer runs the mobile DigMem application (MoDM), and the PC peer runs the DigMem application. Both applications handle connecting to the network and receiving and processing messages; however, DigMem is composed of an extra component, the user interface. This is so that the results sent back from MoDM can be viewed (see Fig. 1). Below is 
C. Dobbins, M. Merabti, P. Fergus, and D. Llewellyn-Jones, "Capturing and Sharing Human Digital Memories with the Aid of Ubiquitous Peer-to-Peer Mobile Services," in The 10th IEEE International Conference on Pervasive Computing and Communications (PerCom'12), 2012, pp. 64-69.

a description of each component and their associated functions.

\section{A. Network Connection}

The network connection manages the state of the network. This includes starting the P2P network, creating the peer group, connecting the peers to the peer group and finding peers within the network.

\section{B. User Interface}

The user interface of DigMem consists of a simple menu screen that lists a number of services that can be found (see Fig. 2). Upon selection of a service, a message is sent to other peers to locate that particular service. The responding peer sends a reply back, which is processed and displayed.

\section{Connection Handler}

The connection handler listens for any events that happen along the pipe and processes all incoming messages for both applications. The MoDM application processes the arriving message and extracts the command, so that appropriate action can be taken. For example, if the command "Take a photo" is received the handler extracts the command and starts the camera. A photo is sent back, in a response message, to the peer that requested it. The connection handler within DigMem does the same job of processing the incoming response. In the case of receiving the photo, the connection handler gets the message, extracts the photo and saves and displays an acknowledgement.

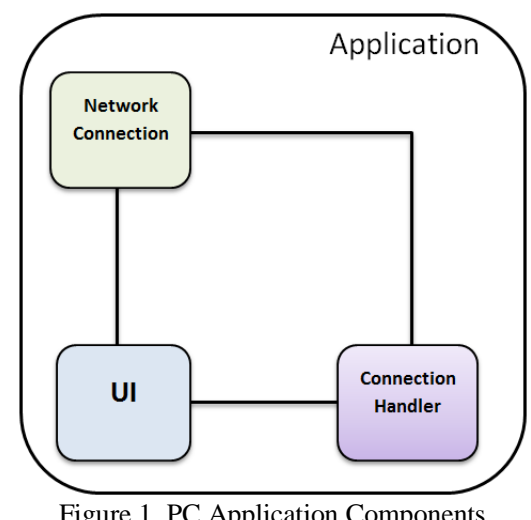

Figure 1. PC Application Components

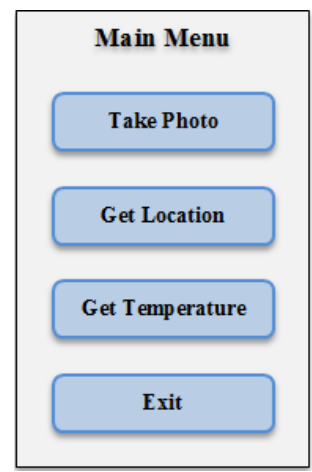

Figure 2. Interface Design
The development of this prototype is the first step in collecting and sharing data, via ubiquitous devices, in order to create human digital memories. By retrieving data from pervasive devices, memories would be richer in their context. Information would be incorporated into the memory that necessarily wouldn't have been available on the current device. For example, if the memory is being created on a PC, this device wouldn't be able to take a temperature reading of the room. However, by connecting to the thermostat this information could be retrieved and sent back, to enhance the memory.

\section{CASe Study}

This section discusses how peer-to-peer mobile services, abstracted from functions provided by different networked devices, can be used to create digital life memories for tracking and identifying sedentary behaviour. In this way, the implementation can be used to demonstrate the case study and test the proposed design. The aim is to establish how data sources can be accessed and used, in order to form part of a digital memory. As well as illustrating how these data sources can underpin digital life memory middleware systems to dynamically discover, compose and adapt collections of data sources in an ad hoc, location and service dependent way.

This system could be used, not only to capture events for reminiscing, but to monitor the health of the user. This case study focuses on using the implemented system to monitor individuals in order to identify and prevent the onset of obesity. The system can be used to identify prolonged periods of sedentary behaviour and markers of a poor diet, which can lead to significant weight gain.

Previous research has indicated that the use of lifelogs provides an accurate track of sedentary behaviour that would otherwise be over-reported by the individual [15]. Monitoring this type of activity, with the use of digital memories can provide a visual illustration of the state of our health and wellbeing, at a particular moment in time. It can also act as a device to predict what our health would be like if we continued on the path we are on [16]. In the scenario of monitoring a user who has a particularly poor diet and is spending prolonged periods in a state of idleness; this could be identified and monitored remotely by their doctor. A request could be made for the use of their camera, which the patient would be wearing, and frequent photos could be taken and sent back for analysis. For a prolonged study, this could indicate that, for example, 10 hours a day the user was in a period of inactivity and that their food consumption was of a relatively poor quality. Over time a significant picture of the user's behaviour would be accumulated and by presenting them with this visual information they could see how this is impacting on their life. 
C. Dobbins, M. Merabti, P. Fergus, and D. Llewellyn-Jones, "Capturing and Sharing Human Digital Memories with the Aid of Ubiquitous Peer-to-Peer Mobile Services," in The 10th IEEE International Conference on Pervasive Computing and Communications (PerCom'12), 2012, pp. 64-69.

The following is the technical description of how the application connects the peers together, in order to obtain the services needed for this scenario.

\section{A. Technical Description}

In order to connect the peers together JXTA [6] has been chosen, as it is compatible with Android and Java. JXTA is an open source platform that is designed to be used by developers, independent of preferred programming languages, development environments, or deployment platforms. It is also implementable on every device with a digital heartbeat [17]. It is for these reasons that the development of the prototype will utilize JXTA.

The demonstration prototype includes three separate peers: a Rendezvous peer, which acts as a "meeting point" for other peers; a PC peer, which discovers data sources and constructs the digital life memories; and a mobile peer, which provides services or data sources.

The following is a description of the sequence for sending a photo request, capturing the photo, and sending it back to the PC peer (see Fig. 3).

1. The rendezvous peer initializes and connects to the JXTA network and becomes a member of the default NetPeerGroup.

2. The mobile peer then starts, connects to JXTA, becomes part of the same peer group and connects to the rendezvous peer.

3. The mobile peer publishes its various advertisements, needed in order to advertise the available services. It then waits for a connection on its server pipe.

4. The PC peer then starts, connects to JXTA, becomes part of the same peer group and connects to the rendezvous peer.

5. The PC peer sends out a discovery message, via the Camera Peer Discovery Handler, in order to find all peers with the specific Module Specification Advertisement of "JXTASPEC:CAMERA".

6. Once the advertisement has been found, the PC peer starts its discoveryEvent(DiscoveryEvent ev) method. This method extracts the Pipe Advertisement, from the discovered Module Specification Advertisement.

7. The createOutputPipe(PipeAdvertisement pipeadv) method is then called. This method creates the output bidirectional pipe, based on the extracted pipe advertisement of the mobile peer from step 6 .

8. The output pipe connects to the mobile peer's waiting server pipe and a set of bidirectional pipes are created and connected for communication.

9. The mobile peer starts its connectionManager thread, which starts the ConnectionHandler class. The
ConnectionHandler implements the PipeMsgListener interface, a the pipeMsgEvent(PipeMsgEvent event) method. This method is started and waits for any incoming messages that have been received along the pipe.

10. Meanwhile, the PC peer makes a call to the sendCommand(JxtaBiDiPipe myOutputPipe) method. This method uses the newly built output pipe in order to send a JXTA message to the mobile peer, containing the command "TakePhoto".

11. Once the message has been sent the PC peer's connectionManager thread is started. This acts as the same as the mobile peer's connectionManager thread and starts the PC peer's own ConnectionHandler class. The PC peer is now waiting for a response from the mobile peer, i.e. the photos captured.

12. The mobile peer receives the message, and via it's ConnectionHandler class starts the pipeMsgEvent(PipeMsgEvent event) method. This method gets the message from the event and extracts the command from the message.

13. Since the command is to take a photo the takePhoto() method is then started. This method uses an Intent to start the CameraFunction class.

14. The CameraFunction class then starts taking the photos, at a fixed interval, and saves them. Photos are taken and saved one at a time and are also sent in this manner as well.

15. Once one photo has been taken and saved a call is made, within the CameraFunction class, to start the ConnectionHandler.sendResponse(ConnectionHandle r.pipe, lastImageSaved) method.

16. The sendResponse(JxtaBiDiPipe pipe, String image) method takes the associated pipe and the newly captured image and creates a new JXTA Message, i.e. the response message. The response message is then sent down the pipe to the PC peer.

17. The photo is then received by the PC peer, via its waiting pipeMsgEvent(PipeMsgEvent event) method.

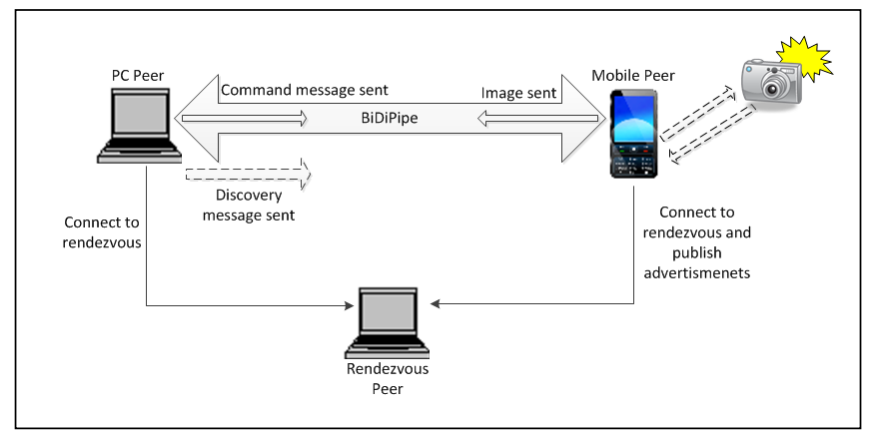

Figure 3. Process of Capturing a Photo 
C. Dobbins, M. Merabti, P. Fergus, and D. Llewellyn-Jones, "Capturing and Sharing Human Digital Memories with the Aid of Ubiquitous Peer-to-Peer Mobile Services," in The 10th IEEE International Conference on Pervasive Computing and Communications (PerCom'12), 2012, pp. 64-69.

Once the PC peer receives the message the photo is extracted and saved into a folder on the PC peer's device. Steps $14-$ 17 are repeated every 10 seconds so that a number of photos are captured and sent back. This sequence of events stops capturing data when the user, in control of the PC peer, deems it necessary.

The implementation of this prototype is the first step in capturing data from ubiquitous devices, in order to build human digital memories. This system paves the way for other forms of data to be discovered and utilized in this way.

\section{Evaluation}

The MoDM application was deployed on a Samsung Galaxy tablet (mobile peer) and DigMem was deployed on a desktop PC (PC peer). The rendezvous peer was also deployed on the PC, as a separate peer. This configuration was used to assess the appropriateness of the proposed design.

The first items to be tested were that the mobile and PC peers could connect to the JXTA network, and that they were all connected to the rendezvous. Once this was established the menu screen, on the PC peer, needed to be verified (see Fig. 4). This was to ensure that when the "Take Photo" button was pressed the discovery message was propagated to all peers on the network.

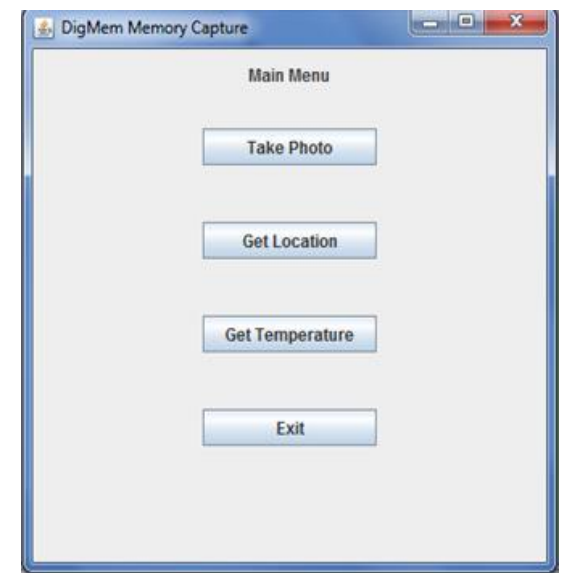

Figure 4. User Interface of DigMem

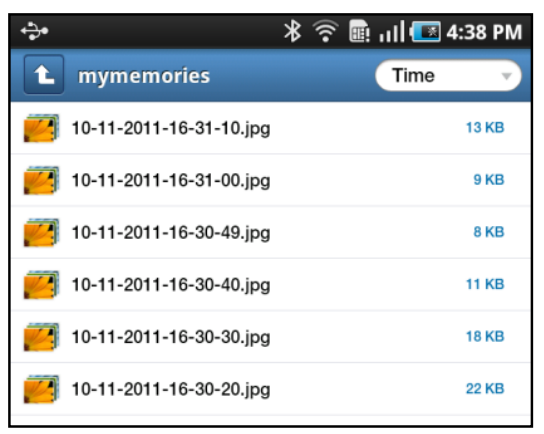

Figure 5. Photos Captured and Saved From the Service, on the Galaxy Device

The next item tested was that the peer with the camera service could be found and that the camera could be automatically triggered, from the command, and the images were being saved (see Fig. 5).

Once this was verified the last item to be tested was that the correct images were being sent back to the PC peer, and that they were being saved (see Fig. 6). The mobile device does not have an interface because, like Microsoft's SenseCam [15]. The photos are taken independently of user interaction. The preview facility isn't required. In order to capture sample data the system was deployed to take photos every 10 seconds, for one minute, (see Fig. 7).

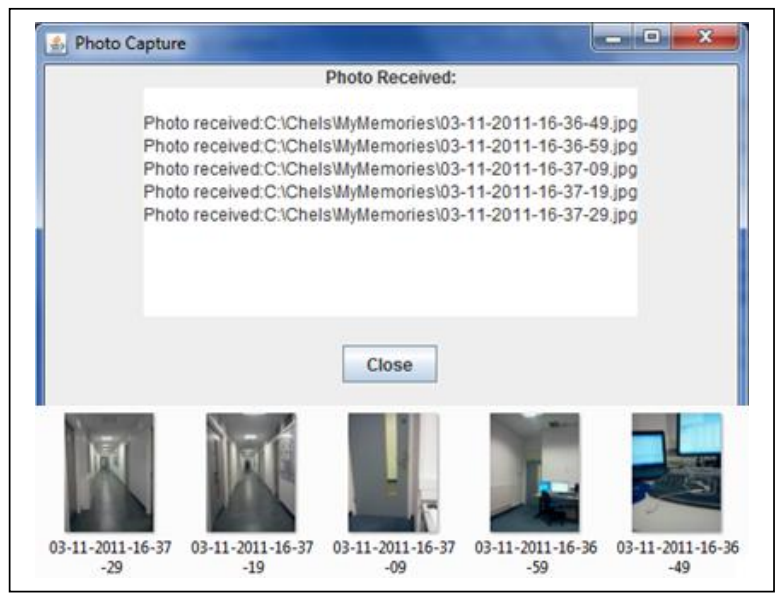

Figure 6. Files Received From the Mobile Peer

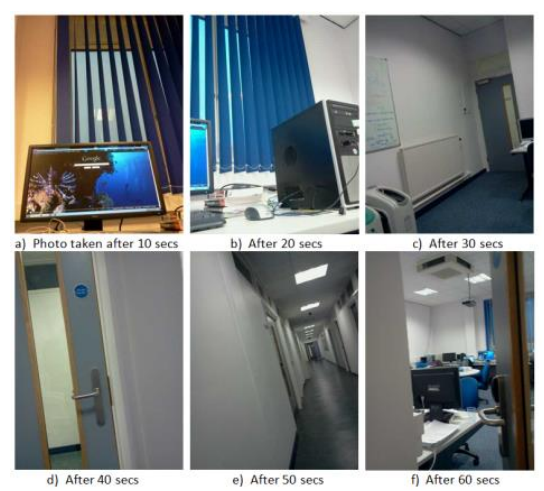

Figure 7. Sample of Photos Taken From the DigMem System

The results illustrate the initial results that were obtained. Although this was a relatively short use of the system, the results indicate that, for extensive use, recording and utilizing data in this way offers a greater insight into capturing our movements. Memories could also be shared among other users in the network, thus building up a picture of the user's day and interactions. By integrating a variety of data sources from ubiquitous devices, this is an advantage that the proposed system has over others, i.e. the SenseCam [18].

\section{CONCLUSION AND FUTURE WORK}

Smart mobile devices are quickly becoming a constant fixture in everyday life. Ofcom's Annual Communications 
C. Dobbins, M. Merabti, P. Fergus, and D. Llewellyn-Jones, "Capturing and Sharing Human Digital Memories with the Aid of Ubiquitous Peer-to-Peer Mobile Services," in The 10th IEEE International Conference on Pervasive Computing and Communications (PerCom'12), 2012, pp. 64-69.

report [19] states "The top three activities/functions ever used on a smartphone (other than making and receiving calls and texts) are internet surfing (69\%), taking photos/videos $(68 \%)$, and email (67\%)". There are also "indications that smartphones are encroaching upon 'traditional' social interaction, with $51 \%$ saying that they ever use their phone while socialising with others". It is clear that capturing and interacting with significant moments is more likely to occur with the assistance of a "smart" device. This technology is the most convenient way to obtain this data and is one that will only strengthen in time.

This paper presents the initial prototype that has been developed in order to capture human memories, with data obtained from ubiquitous mobile devices. Future work aims to develop this prototype further with the addition of more discoverable data sources. By incorporating more information from our surrounding environment and linking them together a "complete" memory is formed. This work will be fundamental in the progression of the development of the system.

Additionally, once the data has been obtained further work can commence into building the memory boxes. The manipulation and interaction of those boxes will then occur. The boxes will be linked, with similar memories being clustered together, forming an endless stream of memories. A rich snapshot of our lives will start to emerge and will be useful in supporting the process of reflecting upon those times and monitoring our wellbeing.

\section{REFERENCES}

[1] M. Parameswaran, A. Susarla, and A. B. Whinston, "P2P Networking: An Information Sharing Alternative," Computer, vol. 34, no. 7, pp. 31-38, Jul. 2001.

[2] Ofcom, "Here are all the latest facts and figures about communications in the UK today," Facts \& Figures, 2011. [Online]. Available: http://media.ofcom.org.uk/facts/. [Accessed: Nov-2011].

[3] International Data Corporation (IDC), "IDC - Press Release,” IDC - Press Release, 2011. [Online]. Available: http://www.idc.com/getdoc.jsp?containerId=prUS2296281 1. [Accessed: Nov-2011].

[4] Android.com, "What is Android?," 2011. [Online]. Available:

http://developer.android.com/guide/basics/what-isandroid.html. [Accessed: Sep-2011].

[5] C. Dobbins, M. Merabti, P. Fergus, and D. LlewellynJones, "Towards a Framework for Capturing and Distributing Rich Interactive Human Digital Memories," in 12th Annual Postgraduate Symposium on the Convergence of Telecommunications, Networking and Broadcasting PGNet, 2011.

[6] Sun Microsystems Inc., "JXTA: The Language and Platform Independent Protocol for P2P Networking." [Online]. Available: http://jxta.kenai.com/index.html. [Accessed: Jun-2011].

[7] Project PeerDroid, "PeerDroid: Jxta Peers Running on Android Platform." [Online]. Available: http://code.google.com/p/peerdroid/. [Accessed: Jun2011].

[8] T. Olsson, M. Lehtonen, D. Pavel, and K. VäänänenVainio-Mattila, "User-Centered Design of a Mobile Application for Sharing Life Memories," Proceedings of the 4th international conference on mobile technology, applications, and systems and the 1st international symposium on Computer human interaction in mobile technology - Mobility '07, p. 524, Sep. 2007.

[9] M. Bisignano, G. D. Modica, and O. Tomarchio, "JMobiPeer: a middleware for mobile peer-to-peer computing in MANETs," First International Workshop on Mobility in Peer-to-Peer Systems (MPPS) (ICDCSW'05), 2005.

[10] A. I. Wang and P. N. Motzfeldt, "Peer2Schedule - An Experimental Peer-to-Peer Application to Support Present Collaboration," 2007 International Conference on Collaborative Computing: Networking, Applications and Worksharing (CollaborateCom 2007), pp. 418-425, Nov. 2007.

[11] F. S. Tsai, W. Han, J. Xu, and H. C. Chua, "Design and Development of a Mobile Peer-to-Peer Social Networking Application," Expert Systems with Applications, vol. 36, no. 8, pp. 11077-11087, Oct. 2009.

[12] C. E. Palazzi, A. Bujari, and E. Cervi, "P2P File Sharing on Mobile Phones: Design and Implementation of a Prototype," 2009 2nd IEEE International Conference on Computer Science and Information Technology, 2009.

[13] A. Ismail, M. Merabti, D. Llewellyn-jones, and S. Sudirman, "Identifying Personal Memories and Shared Experience Using P2P Networks," The 10th Annual Conference on the Convergence of Telecommunications, Networking \& Broadcasting (PGNet 2009), Liverpool, UK, 22-23 June 2009, 2009.

[14] A. Ismail, M. Merabti, D. Llewellyn-Jones, and S. Sudirman, "A Framework For Sharing and Storing Serendipity Moments in Human Life Memory," The First IEEE International Conference on Ubi-media Computing (U-Media 2008), Lanzhou University, China, 15-16 July 2008, pp. 132-137, Jul. 2008.

[15] P. Kelly, A. Doherty, E. Berry, S. Hodges, A. M. Batterham, and C. Foster, "Can We Use Digital Life-Log Images To Investigate Active And Sedentary Travel Behaviour? Results From A Pilot Study," The International Journal of Behavioral Nutrition and Physical Activity, vol. 8, no. 1, p. 44, Jan. 2011.

[16] C. Dobbins, P. Fergus, M. Merabti, and D. LlewellynJones, "Monitoring and Measuring Sedentary Behaviour with the Aid of Human Digital Memories," in 2nd IEEE International Workshop on Consumer eHealth Platforms, Services and Applications - CCNC'2012 CeHPSA Workshop, 2012, pp. 415-418.

[17] L. Gong, "JXTA: A Network Programming Environment," IEEE Internet Computing, vol. 5, no. 3, pp. 88-95, 2001.

[18] S. Hodges et al., "SenseCam: A Retrospective Memory Aid," UbiComp 2006: Ubiquitous Computing, vol. 4206, pp. 177-193, 2006.

[19] Ofcom, "Ofcom Communications Market Report: UK," 2011. 